\title{
The Impact of Prescribed Dosage Assumptions on the Evaluation of Adherence and Persistence to Medication in Patients After Acute Myocardial Infarction
}

\author{
Artis LUGUZIS ${ }^{\mathrm{a}}$ and Juris BARZDINS ${ }^{\mathrm{b}, 1}$ \\ ${ }^{a}$ University of Latvia, Faculty of Physics, Mathematics and Optometry \\ ${ }^{\mathrm{b}}$ University of Latvia, Faculty of Medicine
}

\begin{abstract}
Medication adherence is a significant problem in public health. Prescription-level pharmacy databases have great potential for monitoring actual drug adherence patterns at the healthcare system level. Many research papers have reported adherence estimates in different settings and populations. However, comparison between studies is not always straightforward due to different approaches taken when computing adherence. A crucial component to accurately estimate adherence is the availability of days' supply information for each dispensing event. Reasonable assumptions regarding medication dosage have to be made, when this information is not available. In this study, we evaluate adherence and persistence to medication in patients after myocardial infarction and show that corresponding estimates differ significantly, when using different dosage assumptions, namely, when using defined daily dose or tablet per day dosage regimens. Moreover, we demonstrate that observed differences between medications might be a result of inaccurate dosage assumptions. We propose a comparison of distribution of days between dispensing events to that of days' supplied as a relatively simple visual inspection to validate dosage assumptions.
\end{abstract}

Keywords. adherence, medication dosage, electronic health records

\section{Introduction}

National pharmacy level data submitted for reimbursement purposes allows monitoring actual medication adherence patterns at the whole system level. However, prescription data collected for administrative purposes often lacks prescriber recommendations on medication regimen in each particular case. Therefore, assumptions have to be made.

For cases where days supplied is not available in the database, it is suggested that researcher estimate the days' supply for each drug by applying the defined daily dose to the quantity dispensed [1]. Use of defined daily dose, tablet per day, and three different percentiles of calculated prescribed daily doses have been compared by Ihle et al. [2]. Our study investigates two of these approaches, namely, use of the defined daily dose

${ }^{1}$ Juris Barzdins, Faculty of Medicine, University of Latvia, Jelgavas Street 3, Riga, Latvia; E-mail: juris.barzdins@lu.lv. 
(DDD) proposed for statistics methodology by WHO [3] and tablet per day dosage regimens. We reason that the latter might be the preferred prescription regimen that clinicians choose, if possible, because it is the most convenient for the patient. We offer a relatively easy way to visually inspect validity of chosen dosage assumption and compare how this choice affects both estimated adherence and persistence, following the suggestion to investigate multiple components of adherence by Vrijens et al. [4].

\section{Methods}

To analyze the impact of prescribed dose assumptions, Latvian nationwide pharmacy level data submitted for reimbursement purposes for the period from 2014 to 2018 , linked to the hospitalization events database, was used. All patients discharged alive after the hospitalization with acute myocardial infarction with ST-segment elevation (STEMI) were selected as a subgroup with generally standardized recommendations for further pharmaceutical therapy. The assumption, based on respective European Society of Cardiologists Guidelines [5], was made that most of the patients in 12 months following a discharge should use 1) angiotensin-converting enzyme inhibitors (ACEi) or angiotensin receptor blockers (ARBs), 2) beta-blockers, 3) statins, and 4) platelet inhibitors. Platelet inhibitors generally have prescribed doses equal for all of patients. In contrast, the dosage for the medications belonging to other groups is often case-specific. Thus in our study we analyzed dosage assumptions for ACEi/ARBs, beta-blockers and statins. Analysis was conducted for each medication group separately.

Table 1. Flow diagram of patient selection.

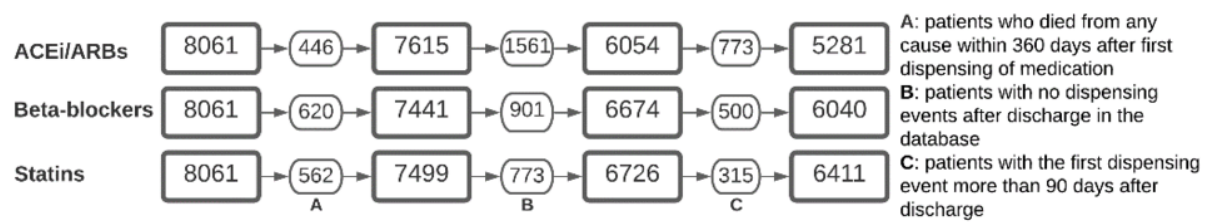

Among 8061 patients discharged alive from a hospital, only patients that had their first dispensing event within 90 days after discharge and were alive 360 days afterward were included in the analysis (Table 1). There were 281609 dispensing events in the database for these patients, 129878 of those in the 360-day observation period.

The database provided information on medications dispensed, including the number of tablets, strength of each tablet in milligrams and Anatomic Therapeutic Chemical (ATC) system code for each dispensing event, however the number of days' supply was not available. We obtained the corresponding DDD in milligrams for each dispensing using the ATC code. As medications may come in various strengths even for the same medication group, we determined for each dispensing the number of defined daily doses in a tablet (DDD/tablet) by dividing the strength of the tablet by DDD. We then computed how frequently each DDD/tablet ratio is encountered in the dataset for each medication group (ACEi and ARBs were analyzed separately here).

We further calculated days' supply with two approaches - setting days' supply equal to number of tablets (i.e. tablet/day regimen) or dividing total quantity of medication dispensed in milligrams by DDD (i.e. DDD/day regimen). In order to validate the two approaches, we compared the distribution of days between subsequent dispensing events with distributions of days' supply computed with tablet/day or DDD/day doses. 
We computed the adherence for the 360-day period using the continuous measure of medication availability (CMA), corresponding to version 6 proposed by Dima et.al. [6]. More precisely, we defined adherence as the proportion of days with medication available within the 360-day period starting at the day of the first dispensing event after discharge from hospital.

We defined persistence as the time to (early) discontinuation of medication [4]. However, because our database is finite, we could not be sure that the patient did not reinitiate medication usage. The operationalization employed was to set the medication discontinuation date as the end date of the last medication usage episode (i.e. when all medication available has been used) within the 360-day observation period. We censored the discontinuation date if the time from discontinuation until the end of the observation period was less than 60 days, as less than $20 \%$ of usage gaps after which medication was reinitiated were longer than 60 days (within our database). Practically, it meant that discontinuation was evaluated only up to day 300 .

We used medians (IQR) to summarize and compare distributions between groups numerically or boxplots for visual comparison.

Data cleaning and processing was performed in R using the tidyverse package [7]. Calculation of adherence and discontinuation was done using functions from the AdhereR package [6].

\section{Results and discussion}

\subsection{Relationship between DDD and tablet}

Table 2 shows DDD/tablet ratios ordered by their frequency (only ratios with frequencies $>0.5 \%$ ). Differences between medications in DDD/tablet ratios can be observed. The majority of ACEi/ARBs and statins are dispensed as tablets with strengths larger or equal to DDD, while strengths of beta-blockers' and platelet inhibitors' tablets are usually less or equal to DDD. This implies that days' supplies, and consequently adherences, will usually be higher using DDD than tablet/day for ACEi/ARBs and statins, but lower for beta-blockers and platelet inhibitors.

Table 2. DDD/tablet (D/t) ratio frequencies by medication. Ratios arranged by relative frequency (\%). Only ratios with frequency $>0.5 \%$ are displayed.

\begin{tabular}{|c|c|c|c|c|c|c|c|c|c|c|c|c|c|c|c|}
\hline \multicolumn{4}{|c|}{ ACEi } & \multicolumn{4}{|c|}{ Beta-blockers } & \multicolumn{4}{|c|}{ Statins } & \multicolumn{4}{|c|}{ ARBs } \\
\hline $\mathrm{D} / \mathrm{t}$ & $\mathbf{N}$ & $\%$ & cum, \% & $\mathrm{D} / \mathrm{t}$ & $\mathbf{n}$ & $\%$ & cum, $\%$ & $\mathrm{D} / \mathrm{t}$ & $\mathbf{n}$ & $\%$ & cum, $\%$ & $\mathrm{D} / \mathrm{t}$ & $\mathbf{n}$ & $\%$ & cum, $\%$ \\
\hline 1.25 & 22184 & 32,9 & 32,9 & 0.5 & 24316 & 28,7 & 28,7 & 4 & 31831 & 42,8 & 42,8 & 2 & 8272 & 65,6 & 65,6 \\
\hline 2 & 14408 & 21,3 & 54,2 & 0.33 & 20387 & 24,0 & 52,7 & 2 & 24172 & 32,5 & 75,3 & 1 & 4029 & 32,0 & 97,6 \\
\hline 1 & 12165 & 18,0 & 72,2 & 1 & 20068 & 23,7 & 76,3 & 1 & 12822 & 17,2 & 92,5 & 0.5 & 191 & 1,5 & 99,1 \\
\hline 2.5 & 11728 & 17,4 & 89,6 & 0.17 & 7413 & 8,7 & 85,1 & 0.5 & 2425 & 3,3 & 95,8 & & & & \\
\hline 4 & 2455 & 3,6 & 93,2 & 0.67 & 7036 & 8,3 & 93,4 & 3 & 1628 & 2,2 & 98,0 & & & & \\
\hline 1.33 & 1744 & 2,6 & 95,8 & 0.25 & 5434 & 6,4 & 99,8 & 1.5 & 1387 & 1,9 & 99,8 & & & & \\
\hline 1.6 & 881 & 1,3 & 97,1 & & & & & & & & & & & & \\
\hline
\end{tabular}

In Figure 1 the distribution of days between subsequent dispensings is compared with distributions of days' supplies computed using either DDD/day or tablet/day. Distributions of days between dispensings and days' supplied should be similar, when patients are mostly adherent and dispensing events take place when previous medication 
has been used. Thus, we might reason that in most cases tablet/day seems to be a more realistic dosage regimen than $\mathrm{DDD} /$ day.

Days between dispenses 追 Days dispensed (DDD/day) 追 Days dispensed (tablet/day)
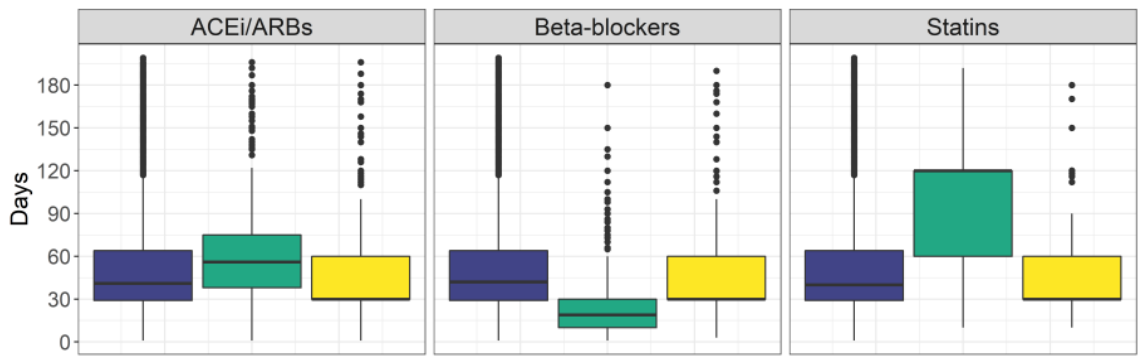

Figure 1. Distributions of days between dispensing events and days' supplied.

\subsection{Adherence}

Distributions of patient adherence values for each medication, computed using both DDD/day and tablet/day regimens are shown in Figure 2. Immediate observation from Figure 2 is that distributions of adherence values seem very similar, when dosage regimen tablet/day is used, more specifically, median adherence among all patients are $66 \%$ (IQR 39\% to 90\%) for ACEi/ARBs, 68\% (IQR 42\% to 93\%) for beta-blockers, and $67 \%$ (IQR $36 \%$ to $88 \%$ ) for statins. As expected, adherences are higher when computed with DDD/day for ACEi/ARBs, 85\% (IQR 50\% to 100\%), and statins, $100 \%$ (IQR 83\% to $100 \%$ ), but lower for beta-blockers, $31 \%$ (IQR $17 \%$ to $50 \%$ ).

Dose 追 DDD/day 追 tablet/day



Figure 2. Distributions of estimated patient adherences by medication.

\subsection{Persistence}

Figure 3 shows survival curves representing the number of patients still using each medication at the given day after first dispensing. Flat segments at the end of each curve are due to censoring discontinuation events after day 300. As the discontinuation date depends on days' supply in the last dispensing event, discontinuation curves also depend on the dosage regimen. As with adherence, persistence patterns are very similar when using tablet/day regimen. Observed proportions of persistent patients at day 180/300 were $79 \% / 65 \%$ for ACEi/ARBs, $80 \% / 67 \%$ for beta-blockers, and $77 \% / 62 \%$ for statins. 
While using DDD/day dosage the corresponding numbers were $82 \% / 72 \%$ for ACEi/ARBs, $77 \% / 60 \%$ for beta-blockers, and $89 \% / 82 \%$ for statins.

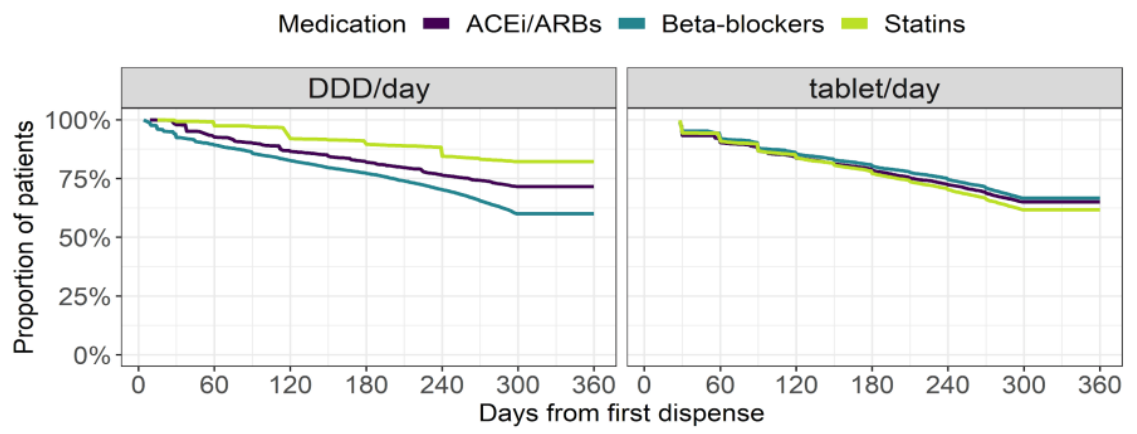

Figure 3. Persistence survival curves by medication.

\section{Conclusions}

Assumptions about dosage or days' supply for each medication dispensing event need to be made, when the underlying pharmacy dispensings' database does not contain such information. This national validation study for two approaches of choosing the appropriate dosage for adherence and persistence calculations, showed that the resulting estimates strongly depend on dosage regimen assumptions. These assumptions may not only bias medication adherence estimates for each particular medication separately, but also the comparison between multiple medications, as adherence might be biased in different directions. Our study suggests that DDD, which is described by WHO as primarily a "unit of measurement and does not necessarily correspond to the recommended or Prescribed Daily Dose" [3], should be used with caution in adherence calculations.

\section{References}

[1] Peterson AM, Nau DP, Cramer JA, Benner J, Gwadry-Sridhar F, Nichol M. A checklist for medication compliance and persistence studies using retrospective databases. Value in Health. 2007;10(1):3-12.

[2] Ihle P, Krueger K, Schubert I, Griese-Mammen N, Parrau N, Laufs U, Schulz M. Comparison of different strategies to measure medication adherence via claims data in patients with chronic heart failure. Clinical Pharmacology \& Therapeutics. 2019;106(1):211-8.

[3] WHO Collaborating Centre for Drug Statistics Methodology. International language for drug utilization research (ATC/DDD), 2020.

[4] Vrijens B, De Geest S, Hughes DA, Przemyslaw K, Demonceau J, Ruppar T, Dobbels F, Fargher E, Morrison V, Lewek P, Matyjaszczyk M. A new taxonomy for describing and defining adherence to medications. British Journal of Clinical Pharmacology. 2012;73(5):691-705.

[5] Ibanez B, James S, Agewall S, Antunes MJ, Bucciarelli-Ducci C, Bueno H, et al. 2017 ESC Guidelines for the management of acute myocardial infarction in patients presenting with ST-segment elevation. European Heart Journal. 2018;39(2):119-77.

[6] Dima AL, Dediu D. Computation of adherence to medication and visualization of medication histories in $\mathrm{R}$ with AdhereR: Towards transparent and reproducible use of electronic healthcare data. PloS One. 2017;12(4):e0174426.

[7] Wickham H, Averick M, Bryan J, Chang W, McGowan LD, François R, Grolemund G, Hayes A, Henry L, Hester J, Kuhn M. Welcome to the Tidyverse. Journal of Open Source Software. 2019;4(43):1686. 